\title{
Evaluating the Multitarget Effects of Combinations through Multistep Clustering of Pharmacological Data: the Example of the Commercial Preparation lberogast
}

\section{(ㄷ) (i) (오 $\ominus$}

\author{
Authors \\ Heba Abdel-Aziz ${ }^{1}$, Olaf Kelber ${ }^{1}$, Gerhard Lorkowski², Martin Storr ${ }^{3}$ \\ Affiliations \\ 1 Phytomedicines Supply and Development Center, Steiger- \\ wald Arzneimittelwerk GmbH, Bayer Consumer Health, \\ Darmstadt, Germany \\ 2 GL Pharma Consulting Research \& Development, Gauting, \\ Germany \\ 3 Center of Endoscopy, Starnberg and Medical Clinic II, \\ Ludwig-Maximilians University, Munich, Germany
}

Key words

cluster analysis, functional dyspepsia, herbal combination preparation, irritable bowel syndrome, multitarget

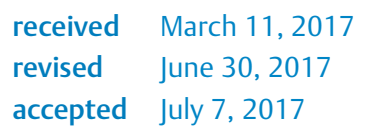

$\circledast$ Supporting information available online at http://www.thieme-connect.de/products

\begin{abstract}
Herbal combination preparations are widely used in traditional herbal medicine and are even established as modern evidence-based herbal medicinal products. The rationale behind such combinations is often questioned and assessing the contribution of each of the combination partners to overall activity is challenging. STW 5 (Iberogast) is such a combination with confirmed clinical efficacy in functional gastrointestinal disorders. It consists of nine plant extracts responsible for its multitarget function in these multifactorial diseases with their heterogeneous and overlapping pathomechanisms. This makes the combination an ideal candidate for the use of the newly described method of stepwise cluster analysis, a standardized procedure to transfer heterogeneous pharmacological data, from different models, into effect size categories. This allows for a stepwise cluster formation starting from the level of single tests up to the level of different pathomechanisms involved in the development of a certain disease, in this case functional dyspepsia subtypes and irritable bowel syndrome. In the current article, an overview on the pharmacological data on STW 5 and its single components is provided. The data are further analyzed using stepwise cluster formation, resulting in a summary of the different modes of action of STW 5 along with an evaluation of the contribution of the single constituents to the overall multitarget effects of the herbal combination preparation.
\end{abstract}

\section{Introduction}

Herbal combination preparations are popular within traditional herbal medicine. They are found in traditional Chinese medicine, Japanese Kampo medicine, Ayurveda, traditional European phytomedicine, etc., as well as in modern evidence-based herbal medicinal products (HMP).

The rationale behind such combinations is frequently questioned and it remains challenging to assess the individual contribution of each of the combination partners to the overall activity of the preparation. This holds especially true when the preparation is used in the treatment of a chronic multifactorial disease as is frequently the case with the most successful HMPs.

STW 5 (Iberogast) is such a combination-preparation with proven clinical efficacy in the treatment of functional gastrointestinal diseases (FGIDs)-a multifactorial set of disorders [1,2]. Over the last 20 years, pharmacological mechanisms of action were characterized for STW 5, as well as for its individual constituents, all probably responsible for its clinical efficacy. STW 5 consists of nine extracts ( $\vee$ Table 1 ) and is used in the treatment of the het- 
- Table 1 Individual extracts of the herbal preparation STW 5 used in pharmacological investigations included in this stepwise cluster analysis.

\begin{tabular}{|l|l|l|l|c|}
\hline Botanical Name & Extract Solvent (V/V) & Drug-Extract Ratio & $\%$ in $\mathbf{1 0 0}$ mL Iberogast \\
\hline Iberis amara L. & Plant Part & $50 \%$ Ethanol & $1: 1.5-2.5$ & 15 \\
\hline Mentha piperita L. & Dresh whole plant & $30 \%$ Ethanol & $1: 2.5-3.5$ & 5 \\
\hline Matricaria chamomilla L. & Dried flowers & $30 \%$ Ethanol & $1: 2-4$ & 20 \\
\hline Glycyrrhiza glabra L. & Dried roots & $30 \%$ Ethanol & $1: 2.5-3.5$ & 10 \\
\hline Angelica archangelica L. & Dried roots & $30 \%$ Ethanol & $1: 2.5-3.5$ & 10 \\
\hline Carum carvi L. & Dried fruits & $30 \%$ Ethanol & $1: 2.5-3.5$ & 10 \\
\hline Silybum marianum L. Gaertn. & Dried fruits & $30 \%$ Ethanol & $1: 2.5-3.5$ & 10 \\
\hline Melissa officinalis L. & Dried leaves & $30 \%$ Ethanol & $1: 2.5-3.5$ & 10 \\
\hline Chelidonium majus L. & Dried herbs & $30 \%$ Ethanol & $1: 2.5-3.5$ & 10 \\
\hline aplant names have been checked on: http://www.theplantlist.org./ & & \\
\end{tabular}

erogeneous and overlapping FGIDs, which makes it an outstanding candidate to present a method to handle complex pharmacological data for multicomponent HMPs with multitarget effects.

FGIDs are the most frequent gastrointestinal disorders in the general population with a prevalence ranging up to $25 \%$. FGIDs are recognized by morphological and physiological abnormalities, which largely occur in combination, including motility disturbances, visceral hypersensitivity, altered mucosal and immune function, altered gut microbiota, and altered central nervous system processing [3].

The most common and best-characterized FGID is irritable bowel syndrome (IBS), where abdominal pain is associated with altered bowel habits like diarrhea (IBS-D), constipation (IBS-C), or alternation between both (IBS-M) [4].

IBS has high prevalence, can significantly impact a patient's function and quality of life, and lacks accepted structural or biochemical abnormalities that may be detected with current routine diagnostic tools [5,6]. Although the underlying pathogenesis is not fully understood, etiological factors include increased epithelial permeability, dysbiosis, inflammation, visceral hypersensitivity, genetic predisposition, and alterations in brain-gut interactions.

The initial treatment strategy is based on predominant symptoms and includes antispasmodics for abdominal pain, antidiarrheal for IBS-D, and laxatives for IBS-C [5]. According to the German treatment recommendations, STW 5 may be recommended as evidence-based treatment for all IBS subtypes for the management of pain, diarrhea-predominant IBS-D, and constipation-predominant IBS-C [7].

Functional dyspepsia (FD) is regarded as a motility disorder dominated by disturbances in gastric physiology. Relaxation of the gastric fundus is impaired and this has been linked to early satiety. Subgroups of FD may exhibit stomach hypersensitivity and disturbed gastric physiology, as well as impaired duodenal motor and sensory functions $[8,9]$.

The revised Rome IV criteria further suggest the subtypes postprandial distress syndrome (PDS) and epigastric pain syndrome (EPS) and conclude that gastroesophageal reflux disease and IBS are part of the FD spectrum [10]. FD is still poorly understood, but recent advances provided new insights into FD pathophysiology, which serves as basis for new treatment options. EPS may be induced by chronic Helicobacter pylori infection in a small subgroup, perhaps via a predominantly antral gastritis that in turn increases gastric acid secretion, resulting in excessive exposition of the duodenum to acid. Acid may in turn activate duodenal sensory nerves and hence induce pain $[10,11]$.

Thus, treatment approaches with antisecretory, spasmolytic, prokinetic, and anti-inflammatory effects and most preferably reduction of visceral hypersensitivity may be useful. This may explain the positive effects of numerous drugs on symptoms and offer a scientifically sound approach for the use of substances, which offer a multitarget action $[11,12]$. STW 5 is such a drug and has been mentioned as a treatment option for both subtypes of FD in the German treatment recommendations, as well as in the Rome IV process [3,7].

STW 5 (Iberogast) is a fixed combination of nine hydroethanolic extracts, namely fresh plant extract from bitter candytuft and drug extracts from peppermint leaves, chamomile flower, liquorice root, angelica root, caraway fruit, milk thistle fruit, lemon balm leaves, and greater celandine herb (further details are presented in $>$ Table $\mathbf{1}$ ).

This paper gives a review of all available pharmacological data on STW 5 and its single extracts in the context of functional gastrointestinal disorders. A method is described to handle this large, complex dataset, first by transferring the results into a standardized categorization of the effect sizes in relation to placebo, controls, or reference drugs. Subsequently, single pharmacological test data were further aggregated by a stepwise cluster formation of single test data in summary tabulations. It is described how pharmacological datasets can be aggregated and structured according to disease-specific symptomatology or postulated pathological mechanisms as shown for the example of STW 5 and FGIDs. Directions and effect sizes of single extracts are compared and evaluated for their contribution to the overall pharmacological activity of STW 5 in FGIDs. 


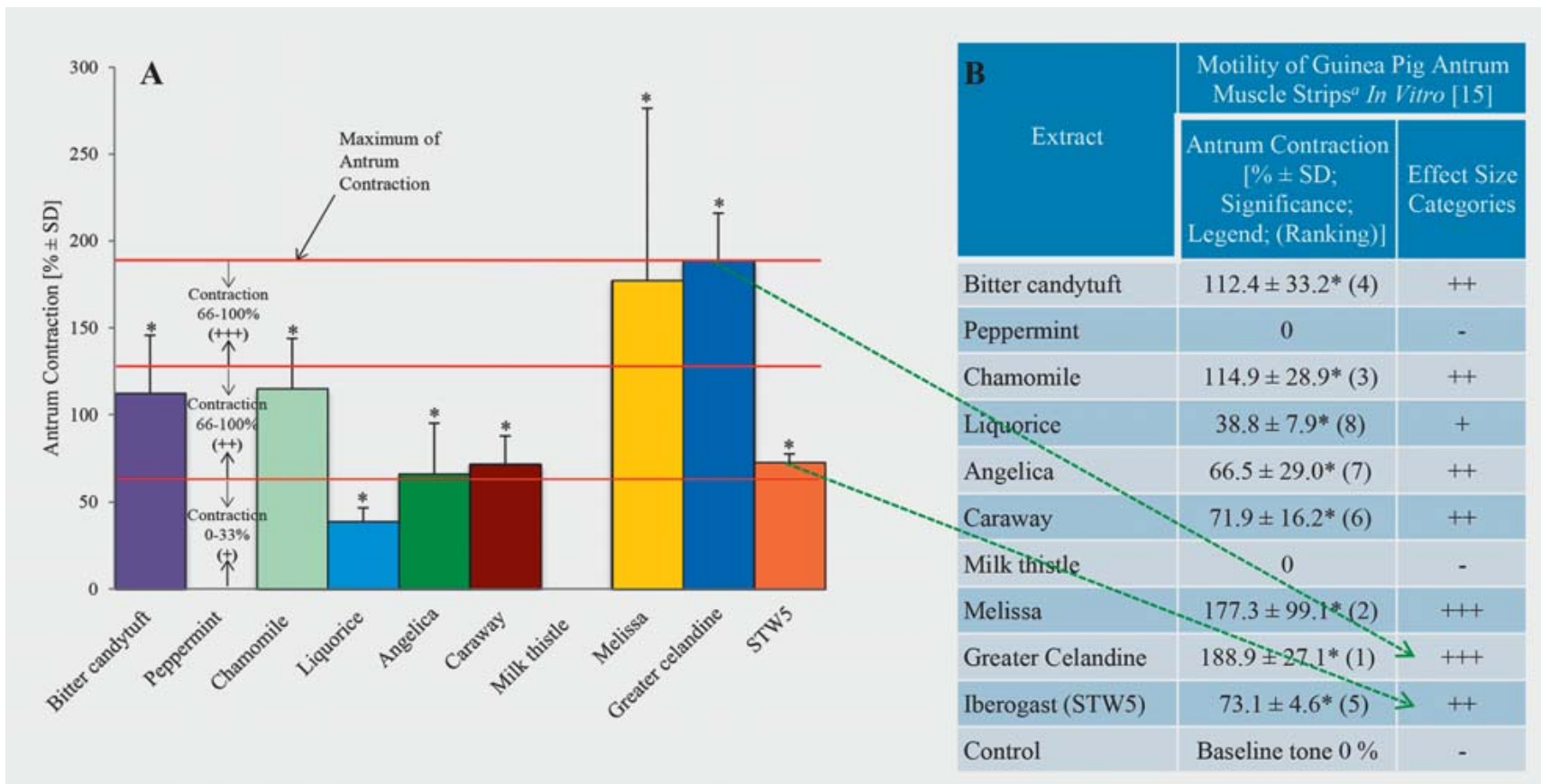

- Fig. 1 Example for data analysis and categorization of pharmacological results. A Standardized categorization of effect size of STW 5 and its single components on contraction of guinea pig antrum muscle strips in vitro compared with control. Shown are results on increased circular and longitudinal muscle contraction by STW 5 and its single constituents compared with control ( $0 \%$ measured in percent (\%) including standard deviation (SD), as well as level of significance $\left.{ }^{*}\right)$. Starting from definition of upper boundary, in this case "maximum antrum contraction" induced by greater celandine ( $189 \%$ corresponding to $100 \%$ effect size) and lower boundary of effect size, $(0 \%$ of control), three effect size categories are defined for the extent of contraction. Effect sizes of 0-33\% are defined as category “+”, of 33-66\% as category “++", and of 66-100\% as category "+++". Effect size categories are then transferred into the summary tabulation (B) as shown for greater celandine and STW 5 (green arrows). B Summary tabulation of data resulting from a single pharmacological test system for STW 5 and its single constituents as well as corresponding effect size categories. Data shown comprise a list of extracts and results from the in vitro measurements of the variable "circular and longitudinal antrum muscle contraction". Effect size was measured in percent (\%) increase of muscle contraction compared to control (0\%). Additionally, SD, significance $\left({ }^{*} \mathrm{p} \leq 0.05\right)$, references to legends, ranking of results for single extracts and STW 5 (numbers in brackets), and dose information for all extracts and STW 5 are given.

\section{Literature Review and Selection}

A PubMed search using the term STW 5 [title] was performed to identify the relevant publications; English and German literature was included. Additional abstracts from scientific data presented at scientific meetings were provided by Steigerwald Arzneimittelwerk GmbH.

The initial review of more than 400 study reports, abstracts, and other publications focused on the selection of a complete set of pharmacological tests performed. Especially those reporting pharmacological investigations using all or selected single plant extracts of STW 5 were of interest. Collection of test systems and characterization of those using single plant extracts may serve to complete or add pharmacological investigations in the future as requested by advances in scientific knowledge. For reasons of clarity and comprehensibility, data were divided the same time in groups according to in vitro test systems (e.g., measurement of antioxidant/radical scavenging activity or specific receptor interactions), species (e.g., mice, rats, guinea pigs, and humans), gastrointestinal localization (e.g., stomach, small, or large intestine), and pathophysiological mechanisms. FGIDs with their overlapping symptomatology and multifactorial genesis (e.g., in- flammation, disturbance of motility, or visceral hypersensitivity) were used as an example to confirm suitability of the method.

Pharmacological data were available for STW 5, several different plant extract combinations, single plant extracts, and controls as well as for respective reference drugs (e.g., cimetidine), system-specific inhibitors to elucidate mechanisms of action, and (toxic) effectors (e.g., acetylcholine [ACh], indomethacin, or 2,4,6-trinitrobenzene sulfonate [TNBS]). Results on plant extract combinations of more than one but not all of the extracts present in STW 5 were excluded from the analyses. Collected data were heterogeneous by corresponding to net values of variables or expression of effects sizes and were not available for all single plant extracts. Collected data represent absolute values, changes from baseline, percent change versus control, or references. Direction of action, level of significance, and ranking of effect sizes could be easily extracted or calculated from ranking of net effects.

- Fig. 1 shows an example for the content of a classical summary tabulation and effect size estimation for the variable antrum muscle contraction, measured as $\mathrm{mN}$ in preparations of circular and longitudinal muscle strips from guinea pig stomach $[13,14]$. Ranking and effect size estimation is generally based on comparison to reference or placebo. If both references are missing, rank- 
ing effects size estimation results from comparison with either the lowest or highest single plant extract effect size as shown in - Fig. $1 \mathrm{~A}$, where maximum of antrum contraction induced by greater celandine is used as reference. Results on direction of action, lack of activity, problems in measurements, or others could easily be included in the summary tabulation's legend ( $\bullet$ Fig. 1 B).

\section{Categorization Process}

Subsequently, a strategy was developed to transfer numeric data into three effect size categories. Negative and opposing results were categorized as "-[0]" and missing investigations as "-" corresponding to "not available." Transfer of data into size categories allows for comparison and pooling of different kinds of pharmacological data. As can easily be seen from • Fig. 1 A, a simple categorization according to ranking of effect size would be misleading. To generate effect size categories from ranking of single extracts relative to chosen reference, they have to be pooled into three categories as shown in $>$ Fig. 1 A. Highest effects sizes represented by ranking 1-3 have to be pooled into category "+++", ranking 4-6 in category "++", and ranking 7-9 in category "+". In this example, STW 5 is not included in the ranking procedure. If necessary, it can be estimated by comparison with single plant extract ranking. According to this definition, in case of antrum contraction chamomile extract (ranking of 3 ) with an about $80 \%$ lower activity is pooled together with the extracts of melissa and greater celandine, which is misleading.

Categorization started with selection of the $100 \%$ activity margin characterized by upper and lower boundaries for the selected variable. In the example shown in - Fig. $\mathbf{1 A}$, increase of antrum contraction to about $190 \%$ of greater celandine was set as upper boundary ( $100 \%$ increase). Except for milk thistle, all single plant extracts increased antrum contraction as shown in > Fig. 1 A. Therefore, the lower boundary of effect size was set to baseline value ( $0 \%$ increase). Categories of increase of antrum contraction are described in this example by the following orders of magnitude of increase: 0-33\%: (+); 34-66\%: $(++)$, and 67-100\%: $(+++)$. Results are shown as effect size categories for increase of antrum contraction in the right column of $>$ Fig. 1 B (green arrows). All effect sizes summarized in summary tabulations for STW 5, single plant extracts, and pharmacological parameters were transferred into three effect size categories according to the method chosen for the respective test system.

Further on, similar pharmacological parameters (e.g., reduction of acid content and output $[15,16]$ as well as histamine- or dibutyryl cyclic adenosine monophosphate (cAMP)-stimulated acid production [17]) were clustered (first order cluster) and mean effect sizes for STW 5, and single plant extracts were calculated based on "plus" and "minus" categorizations $(+=1$; $++=2 ;+++=3)$ and transferred again to a "plus" categorization $(<1.5=$ "+”; 1.50-2.499 = "++"; $\geq 2.50=$ "+++") to allow for a comparison of single plant extracts and STW 5 in the first order cluster.

Acid regulation is not the only risk factor contributing to gastric ulcer formation. Pharmacological parameters like mucin and pepsin concentrations as well as prostaglandins and leukotrienes in the mucosal content are further risk indicators of gastric ulcer formation in response to indomethacin-induced inflammatory processes. Dependent on the direction of effect, either increase or decrease of the amount of investigated variables induced by STW 5 or its single plant extracts is indicative of therapeutic effects. An opposite effect of a single plant extract (e.g., relaxation of intestinal muscle) in a pooled variable "tonicizing effects" is not respected for the calculation of means, but in the pooled variable "spasmolytic effects", it is included. For this pooled variable, tonicizing effects have not been considered. Thus, summary tabulations may serve for further clustering (second order cluster) of pharmacological effects for STW 5 and its single plant extracts from different pharmacological investigations (e.g., contribution to indomethacin-induced gastric inflammation).

This allowed for a meaningful clustering (third order cluster) of single pharmacological parameter from the same or other test systems corresponding to disease-specific symptoms or pathophysiological mechanisms, as described before for gastric inflammation. Clusters of pooled variables of different systems/models (e.g., second order cluster "antioxidant/radical scavenging", second order cluster "spasmolytic effect") have been formed and analyzed as shown in Table 3S (Supporting Information) to further characterize contribution of single plant extracts to anti-inflammatory effects of STW 5.

Finally, a further higher level of pooled clusters (fourth order cluster) from different pharmacological systems/models with respect to disease-specific symptomatology or pathophysiological mechanisms of FGIDs, namely FD (EPS and PDS) and IBS ( $\vee$ Table 2), can be formed to differentiate contribution of STW 5 and its single plant extracts to treatment effects in symptom complexes or diseases. It should clearly be mentioned that the content of pharmacological parameters at the level of each cluster order can be varied by extension or deletion to allow for an evaluation of the contribution of single plant extracts and STW 5 to even overlapping disorders. The clustering process is demonstrated in

- Fig. 2.

\section{Effects of STW 5 on Pathological Mechanisms of FGIDs}

- Table 2 summarizes the different pathomechanisms suggested for FD and IBS. FGIDs not only overlap in symptomatology and prevalence, but also seem to share common pathophysiological mechanisms. STW 5 and its constituents were shown to influence the majority of these mechanisms ( $\bullet$ Fig.3). Detailed tables showing all categorization results for the different models and pathomechanisms are presented as supporting information. In the following we give an overview on the effects of STW 5 and the contribution of its constituents (if available) on each of the debated disease mechanisms.

\section{Lower esophageal sphincter pressure}

Lower esophageal sphincter pressure (LESP) has an important role in acid-related symptoms in FD (e.g., heartburn). Increased LESP may lead to symptom improvement. Treatment of isolated muscle strips of guinea pig LES with STW 5 resulted in dose-dependent increase of basal tonus, whereas the directly adjacent 
- Table 2 Formation of symptom- or pathology-associated clusters of subtypes of functional gastrointestinal disorders.

\begin{tabular}{|c|c|c|}
\hline Functional Dyspepsia/Epigastric Pain Syndrome & Functional Dyspepsia/Postprandial Distress Syndrome & Irritable Bowel Syndrome \\
\hline $\begin{array}{l}\text { Gastric accommodation (contraction/relaxation): } \\
\text { - Fundus/corpus } \\
\text { - Antrum }\end{array}$ & $\begin{array}{l}\text { Gastric accommodation (contraction/relaxation): } \\
\text { - Fundus/corpus } \\
\text { - Antrum }\end{array}$ & $\begin{array}{l}\text { Intestinal motility } \\
\text { - Contraction } \\
\text { - Relaxation }\end{array}$ \\
\hline Visceral hypersensitivity & Visceral hypersensitivity & Visceral hypersensitivity \\
\hline Stomach inflammation & Gastro-duodenal inflammation & Intestinal inflammation \\
\hline Low esophageal sphincter & Mucosa protection & Secretion \\
\hline Acid regulation & $5-\mathrm{HT}_{3}$ receptors (nausea) & Mucosal barrier \\
\hline Mucosa protection & & Microbiome \\
\hline
\end{tabular}

gastric sling muscle and the pylorus remained unaffected indicating specificity to LES (Table 1S, Supporting Information) [18].

\section{Region-specific activity on stomach motility (gastric accommodation)}

There is growing evidence that dysregulation of gastric motility, characterized by impaired gastric relaxation together with antral hypomotility, represents a key mechanism at least in a subset of FD patients. Antral hypomotility may cause delayed gastric emptying while abnormalities in proximal gastric function lead to impaired fundus/corpus relaxation with consecutive hypersensitivity to distension $[14,15]$. Interestingly, STW 5 shows region-specific effects in the stomach, relaxing the fundus and corpus regions while increasing antral contractions, hence counteracting disturbed gastric accommodation seen in FD patients. These effects have been first demonstrated in guinea pig gastric muscle strips ex vivo $[13,14]$ and were later clinically confirmed in volunteers [19]. In order to investigate the contribution of the single extracts to the overall effects of STW 5, further experiments were conducted in guinea pig stomach strips. Only the extracts of angelica $(+++)$, chamomile $(++)$, and liquorice $(+)$ mimicked the relaxing effect of STW 5 in the proximal stomach, while almost all extracts, except peppermint and milk thistle, contributed to the STW 5 effect on antral contractility ( $\bullet$ Figs. 4 and 5, Tables $1 S$ and 2S, Supporting Information) $[13,14]$.

\section{Acid regulation}

Enhanced stomach acidity or hypersensitivity to acid can contribute to FD symptoms. STW 5 has been shown to reduce pathologically increased acid secretion in vivo and in vitro [15-17]. Notably, STW 5 had no effect on physiological, unstimulated gastric acidity $[17,20]$.

In vivo, STW 5 and its constituents have been investigated in male Wistar rats following oral indomethacin administration. While liquorice, peppermint, and milk thistle showed lower activity $(++)$, all other extracts significantly reduced gastric juice acidity and acid output by more than $60 \%\left({ }^{++}\right)$(Table 4S, Supporting Information) $[15,16]$.

In vitro, STW 5 showed strong inhibition $(+++/++)$ of acid secretion from isolated guinea pig parietal cells stimulated with histamine or dibutyryl cAMP. Acid-inhibitory activity shown in vivo could be confirmed in this model for bitter candytuft, pepper- mint, chamomile, liquorice, and greater celandine (Table 4S, Supporting Information) [17].

\section{Mucosal protection}

The mucosal barrier is essential for the protection of the entire gastrointestinal tract against damages caused by external or internal aggressive agents (e.g., drugs, bacterial toxins, acid, pepsin). Several gastrointestinal disorders including FD and IBS have been correlated with mucosal barrier impairment. Mucosal barrier integrity is strongly influenced by $\mathrm{PGE}_{2}$ (PGE: prostaglandin) and mucin secretion from goblet cells. STW 5 was shown to increase both mucin and $\mathrm{PGE}_{2}$ tissue concentrations in indomethacin-challenged rats in vivo, characterizing its mucosal protecting effect. All constituents of STW 5 contributed to enhanced gastric $\mathrm{PGE}_{2}$ content in slightly differing extents $(+++/++)$ (Table 4S, Supporting Information). The same holds true for the increase of mucin content - which was decreased by indomethacin administration back to control levels. However, STW 5 elevated mucin concentrations nearly threefold compared to controls, an effect mainly mediated by milk thistle $(+++)$ and to lesser extents by chamomile, liquorice, and caraway $(++)[15,16]$.

\section{Gastrointestinal inflammation}

Low-grade, chronic gastrointestinal inflammation has been implicated in both FD and IBS. For instance, a subset of IBS patients show increased immune cell infiltration into the gastrointestinal wall and it is estimated that up to $30 \%$ of IBS cases are postinfectious, thus related to inflammation [5]. For FD, chronic $H$. pylori infection may be a contributing factor. Other studies also showed a strong correlation between low-grade gastroduodenal inflammation and FD [21].

Potent anti-inflammatory effects have been shown for STW 5 throughout the gastrointestinal tract, both in vitro and in vivo. Starting at the esophagus, where STW 5 largely attenuated reflux esophagitis in acute and sub-chronic settings [20,22]. In the stomach, STW 5 prevented indomethacin-induced inflammation as confirmed by the reduction of leukotriene concentrations, ulcer indices, and prevention of histologically assessed tissue damage. The latter effects were mediated by all constituents to nearly the same extent $(+++/++)$ ( Fig. 4, Table 4S, Supporting Information) $[15,16]$. 


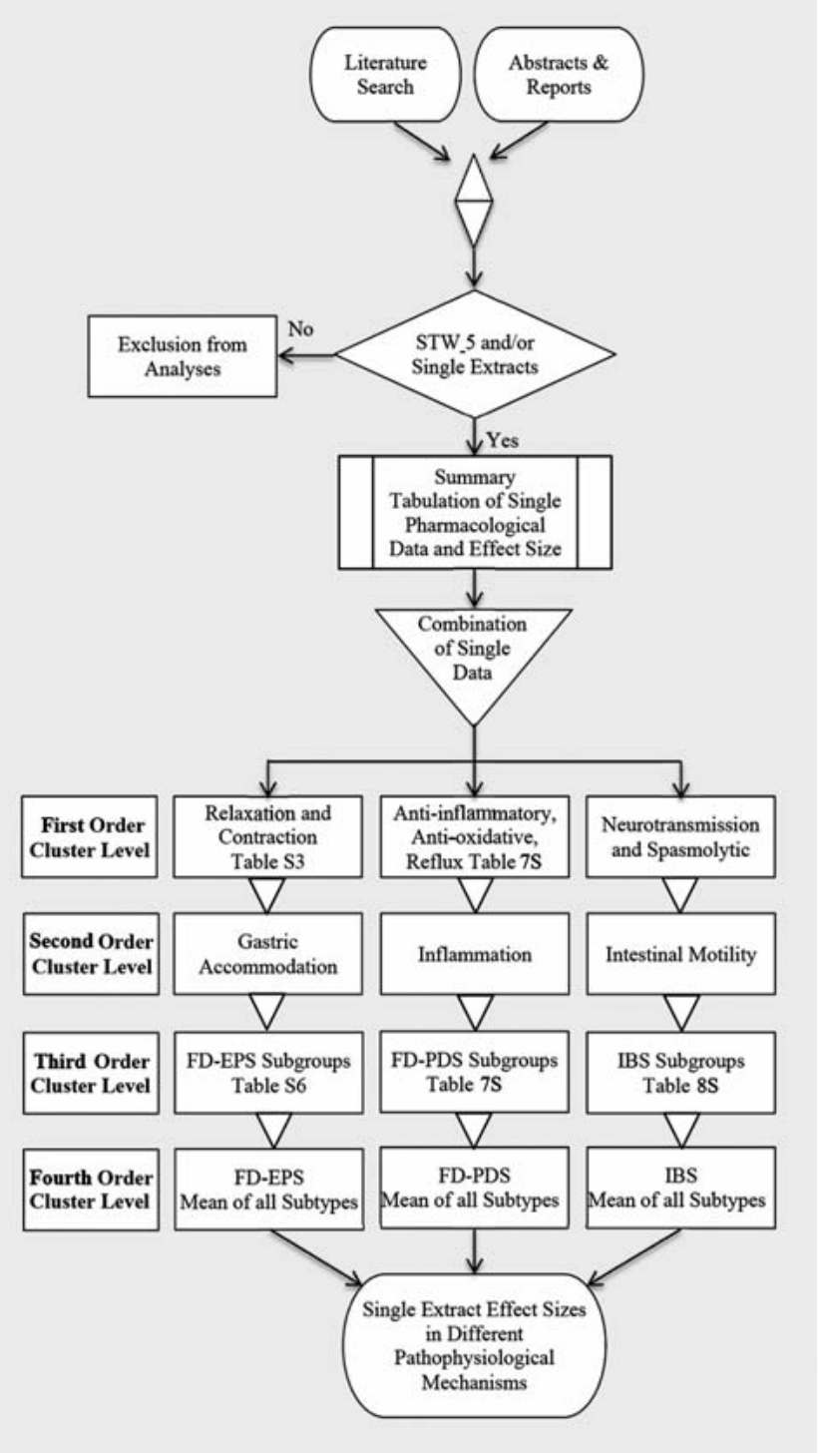

- Fig. 2 Example process of stepwise cluster formation. Publications from a literature search and those from company's database including also abstracts and reports were pooled in the first step and reviewed for availability of data for STW 5 and single plant extracts. In several steps a tabulation of single pharmacological data and corresponding effect sizes was performed considering a variety of subgroups (e.g., laboratory tests, animal systems, location, etc.). All single test data were pooled in the first order cluster level for the two FD subgroups and IBS. These groups were further combined in second order cluster level (gastric accommodation [FD-EPS], inflammation [FD-PDS], or intestinal motility [IBS]). As shown in - Table 1, all subgroups for the FD subgroups and IBS are each combined in third order cluster level as shown in Tables 4S, 5S, and $6 \mathrm{~S}$ (Supporting Information). Overall mean in before-mentioned tables is the fourth order cluster level result for FD-EPS, FD-PDS, and IBS.

As for the lower gastrointestinal tract, several in vitro and in vivo models were used to investigate the effects of STW 5 on small intestinal and colonic inflammation. The different in vitro models showed that the potent anti-inflammatory effects of STW 5 are

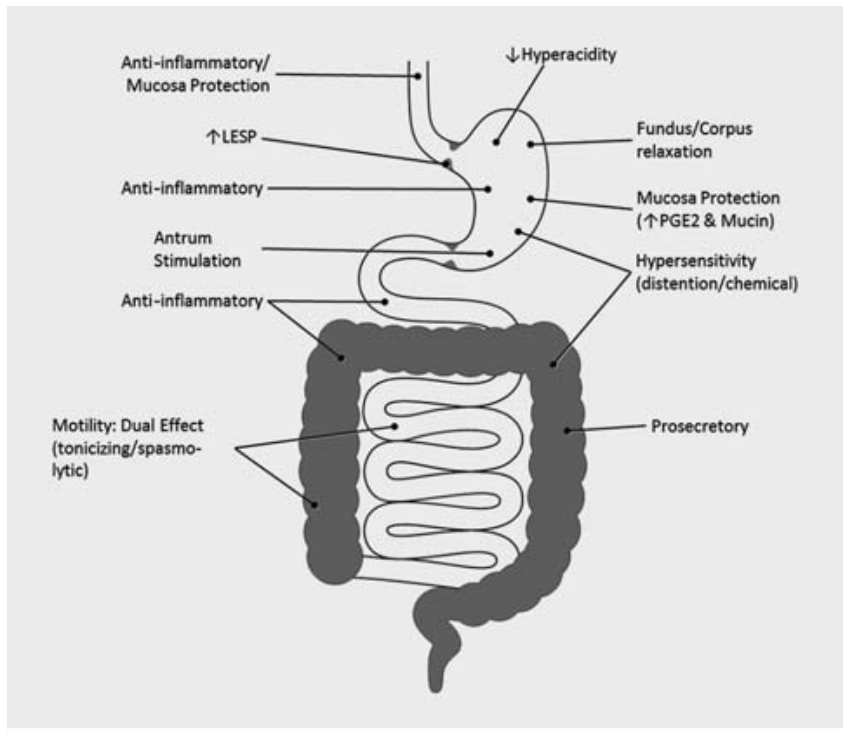

> Fig. 3 Regional pharmacological mechanisms of action of STW 5.

mediated by multiple mechanisms including the activation of adenosine $A_{2 A}$ receptors, the inhibition of pro-inflammatory mediators like TNF- $\alpha$, IL- 8 , and leukotrienes and the enhancement of anti-inflammatory mediators like IL-10. However, STW 5's anti-inflammatory effects do not seem to be mediated by COX inhibition, an effect that contributes to its mucosa-protective activity. All single extracts contributed to the overall anti-inflammatory effect. The strongest actions were seen with melissa followed by bitter candytuft $(++)$ ( ${ }^{+}$Fig. 6, Table 35 and 6S, Supporting Information) [23-33].

Furthermore, STW 5 and its constituents possess potent radical scavenging and anti-oxidant activities (evaluated employing chemical, cell-based and in vivo settings), which contribute to STW 5's anti-inflammatory and mucosa-protective actions [20, 33-35]. While all extracts were involved in the antioxidant effect, supra-additive effects were identified for STW 5 in some of the experimental settings used [35].

\section{Visceral hypersensitivity/serotonin}

Another component of the multifactorial pathogenesis of FGIDs is visceral hypersensitivity. The mechanisms behind visceral hypersensitivity are not completely understood. However, post-inflammatory events seem to play an important role, probably by modulating mechano- and chemo-sensitive afferent neurons, for example those activated by 5-HT (serotonin) or bradykinin.

The beneficial effects of STW 5 on hypersensitivity were shown in small intestine and colon in vivo and ex vivo. For this purpose, rats were pretreated with STW 5 or vehicle. Under anesthesia, mesenteric afferent nerve responses to increasing doses of 5-HT, bradykinin, and ramp distension were quantified in the small intestine in vivo (Table 6S, Supporting Information) [36, 37].

To confirm the in vivo findings-obtained for the small intestine - for the colon, an ex vivo organ bath setting was adopted, in which mesenteric nerve discharge to the same stimuli was recorded in isolated mouse colon. STW 5 and bitter candytuft were 


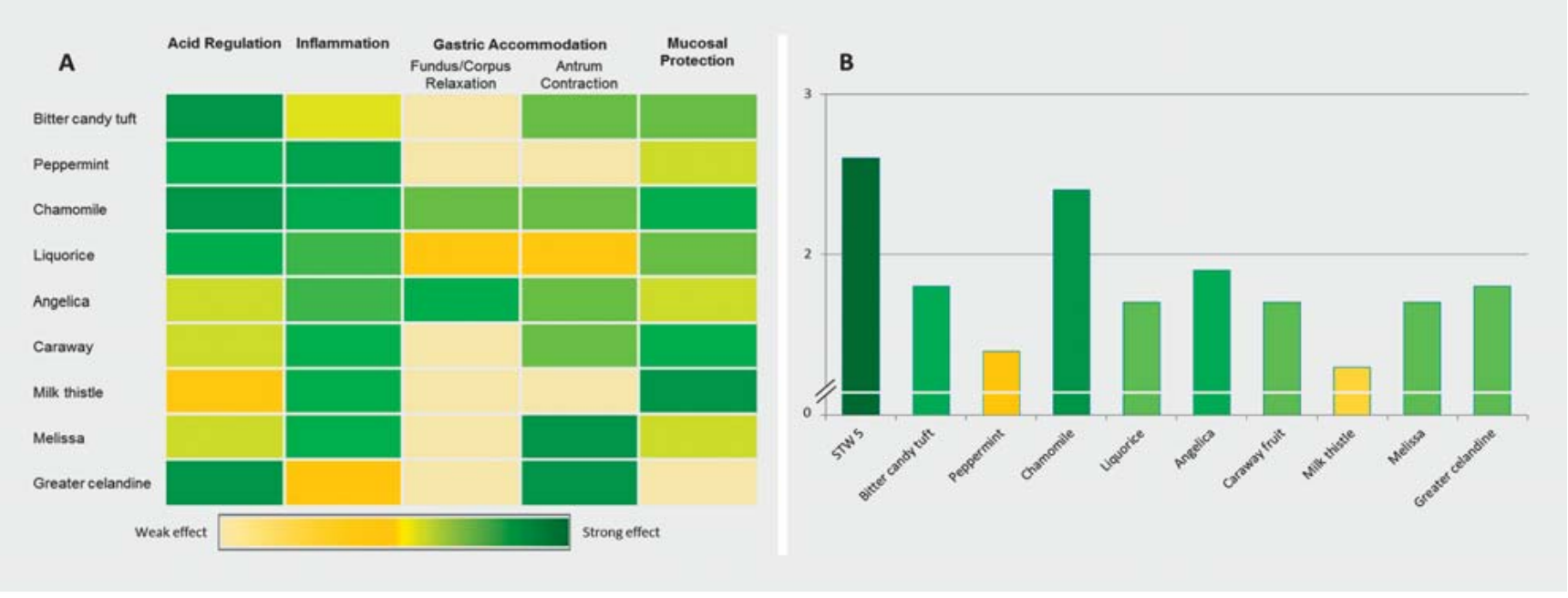

- Fig. 4 Cluster results for the contribution of single extracts to the activity of STW 5 to different pathomechanisms of EPS as a subtype of functional dyspepsia. A Heat-map showing relative effect strength of each extract as color intensity (light yellow to dark green corresponding to 0 to +++ ). B Overall relative activity of STW 5 and its constituents in EPS according to fourth order clustering based on the discussed pathomechanisms represented as mean effect size.

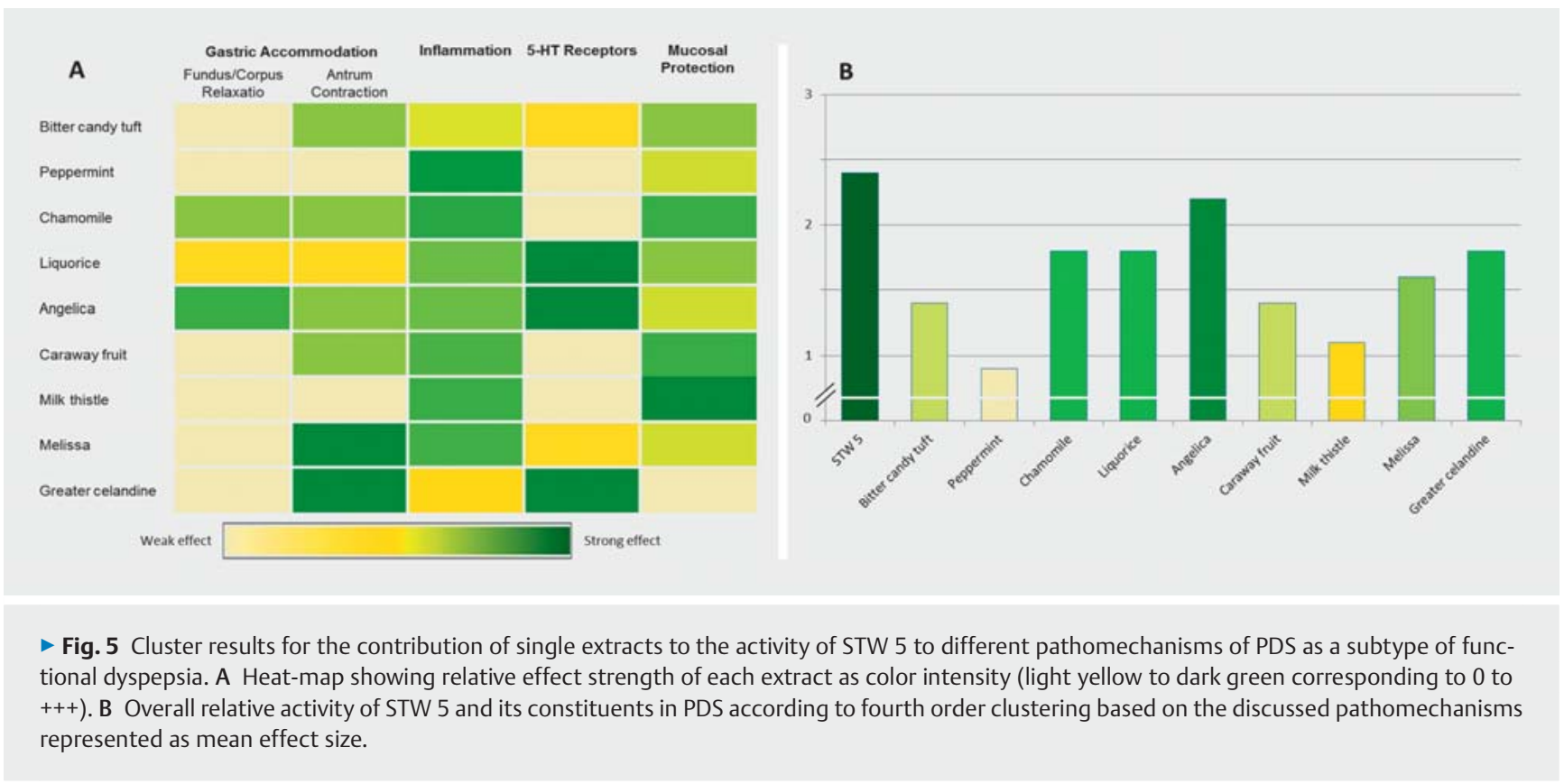

tested and both reduced intestinal afferent nerve discharge in both models. However, bitter candytuft $(++)$ reduced selectively the reactivity of 5-HT-sensitive and low-threshold mechano-sensitive afferents, while STW 5 reduced the responses to all stimuli $(+++)$ and was effective on low- and high-threshold afferents, showing a much broader activity profile (Table 15 and 4S, Supporting Information) [38].

Since $5-\mathrm{HT}$ is one of the main transmitters in the gastrointestinal tract, binding affinities of STW 5 and its constituents were determined for $5-\mathrm{HT}_{3}$ and $5-\mathrm{HT}_{4}$ receptors, the main serotonin receptors in the gut. STW 5 interacted mainly with $5-\mathrm{HT}_{3}$ receptors, with liquorice, angelica, and greater celandine being the main contributors to this effect $(+++)$ ( $\bullet$ Fig. 5, Table 2S, Supporting Information) [39]. STW 5 receptor-blocking activity was further confirmed by measuring $5-\mathrm{HT}_{3}$ receptor-mediated ileal contractions in a functional test in vitro (Table 4S, Supporting Information) [40]. 5- $\mathrm{HT}_{3}$ receptors are also known to play an important role in nausea and vomiting, which may contribute to STW 5's clinical benefits in FD. 


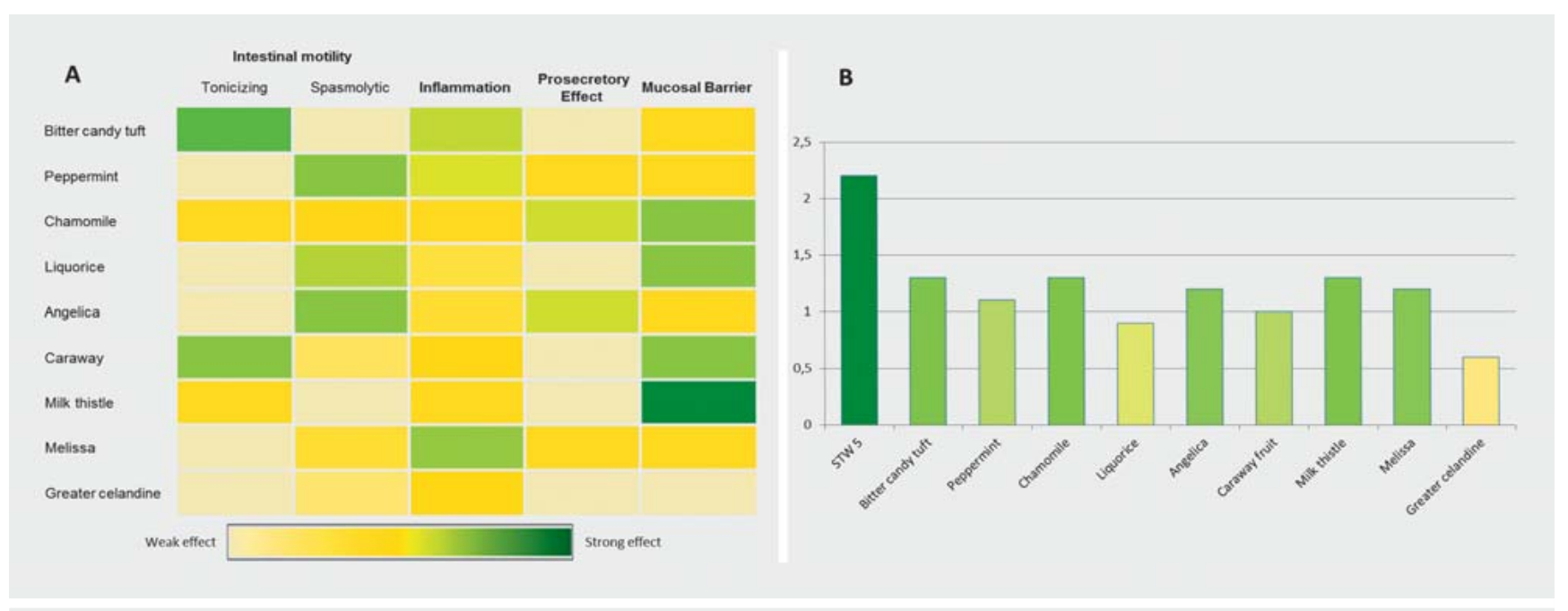

- Fig. 6 Cluster results for the contribution of single extracts to the activity of STW 5 to different pathomechanisms of IBS. A Heat-map showing relative effect strength of each extract as color intensity (light yellow to dark green corresponding to 0 to +++). B Overall relative activity of STW 5 and its constituents in IBS according to fourth order clustering based on the discussed pathomechanisms represented as mean effect size.

\section{Intestinal motility}

IBS is associated with dysregulation of intestinal motility, reflected in altered bowel habits, intestinal spasms, and abdominal pain. Studies showed that patients with IBS, when compared to normal subjects, had an enhanced motor response to different environmental stimuli such as psychological stress, peptide hormones, or fatty meals [1]. Motility can be enhanced or decreased, dependent on the individual patient and the individual IBS subtype. Clinical investigations showed that STW 5 is effective in all IBS subtypes. This can be partly explained by the dual action of STW 5 on intestinal motility. STW 5 acts either by increasing basal contractile tone or by acting spasmolytic, depending on the basal condition of the intestine (eukinetic effect) (i.e., STW 5 contracts atonic muscles but relaxes precontracted ones) [41].

Different extracts contribute to this dual action ( $\bullet$ Fig. 6, Table 3S). Ammon et al. [41] were the first to show that the tonicizing effects of STW 5 in relaxed intestinal muscles (guinea pig ileum) were mainly based on its components bitter candytuft $(++)$ and to a lesser extent on caraway $(++)$. In precontracted ileal segments, STW 5 exerted spasmolytic effects largely mediated by peppermint, angelica, and liquorice [41].

Tonicizing effects were furthermore reported in inflammationinduced intestinal dysmotility. In an experimental model of dextran sulphate sodium (DSS)-induced colitis, pretreatment of rats with STW 5 for one week effectively prevented inflammation-induced decreased colonic motor response to carbachol or $\mathrm{KCl}$ [32]. Voß et al. [26, 27, 42] broadened these findings in an ex vivo model of colitis, where STW 5 normalized TNBS-induced attenuated colonic reactivity to acetylcholine. As described for non-inflamed atonic intestine [41], this tonicizing effect was mainly mediated by bitter candytuft and caraway $(++)$ (Tables 35 and 65 , Supporting Information).

The spasmolytic activities of STW 5 were further characterized in isolated neostigmine-challenged mouse small intestines [30] and in human small and large intestinal tissue preparations [43].
In the latter, STW 5 inhibited both muscle tone and motility index. Contributing extracts were those already identified by Ammon et al. [41], peppermint and liquorice, in addition to angelica, which was not active in Ammon's model (all ++). Effects of lemon balm, caraway, and greater celandine varied according to region [43]. A comparable spasmolytic effect was shown for electrical field stimulation-induced cholinergic contractions (human colon). Single components responsible for inhibitions here were angelica, melissa, and greater celandine [44] (Table 6S, Supporting Information).

\section{Intestinal prosecretory effects}

IBS is a functional bowel disorder characterized by stool irregularities. Therefore, some newly developed drugs target intestinal secretions in order to improve constipation and its related symptoms. Investigations using human intestinal tissues, or the human epithelial cell-line T84, demonstrated that STW 5 possesses prosecretory effects mediated by activation of CAMP- and $\mathrm{Ca}^{++}$-activated. $\mathrm{Cl}^{-}$-channels. In Ussing chamber experiments, the prosecretory effect of STW 5 was due to angelica $(++)$, peppermint $(+)$, and melissa $(+)$. The results were comparable in T84-cells except that angelica had a weaker effect $(+)$ while chamomile showed the strongest prosecretory action (+++) (Table 6S, Supporting Information). Overall, the effects of single extracts were of low activities $(++\mid+)$ and added up to a strong summation effect in STW $5(+++)$ ( Fig. 6, Table 3S, Supporting Information) $[43,45]$.

\section{Functional dyspepsia}

Aside from a patient's predisposition and psychiatric status, inflammation is considered one of the pathomechanisms involved in EPS. An antral predominant gastritis increases gastric acid secretion, resulting in excessive duodenal exposure to acid [8]. This acid overexposition of the duodenum leads to activation of duodenal sensory nerves resulting in pain, symptom generation, and motility changes in FD [46]. Since gastroduodenal inflammation and some other pathomechanisms may be linked to both FD sub- 
types, EPS and PDS, lower level cluster results can be selected and combined as needed for certain pathologies.

Thus, STW 5 and its single plant extracts have been evaluated for their activity in the fourth order EPS cluster for acid regulation and mucosal protection, inflammation, visceral hypersensitivity, and gastroduodenal motility represented by gastric accommodation and LESP. Based on the models used and the available data for single plant extracts for this cluster, STW 5 was found to be the most active preparation $(+++)$, followed by chamomile $>$ angelica $>$ greater celandine, bitter candy tuft > melissa, caraway, and liquorice $(++)$, while peppermint and milk thistle showed the lowest activity $(+)$ ( ${ }^{\circ}$ Fig. 4 B, Table 1S, Supporting Information). However, lacking data for single plant extracts in visceral hypersensitivity and LESP were not considered for calculations of the mean (Table 1S, Supporting Information).

Gastric accommodation is an example showing that combinations are not only valuable in broadening the effects of a preparation - in the sense of a multitarget effect - and because of the generation of additive and synergistic activities, but also because combinations can lead to the elimination of undesirable effects of one or more combination partners. STW 5 was shown to significantly and dose-dependently decrease muscle tone in the gastric fundus and corpus and to enhance antral contractility, which are both desirable effects in FD. All single plant extracts increased antral contractility, except peppermint and milk thistle. Angelica, chamomile, and liquorice extracts decreased muscle tone in fundus and corpus. In contrast, four single plant extracts, namely greater celandine, lemon balm, caraway, and bitter candytuft, significantly enhanced the muscle tone in fundus and corpus. This undesirable effect is counteracted by the combination in STW 5 ( Fig. 4 A, Table 1S, Supporting Information).

Occurrence of symptoms postprandially rather than before or in-between meals is an essential aspect for a diagnosis of PDS, and in contrast to EPS, pain associated with PDS is not required to be meal-related. Additionally, early satiety or postprandial fullness is likely to identify the true PDS subset. Due to overlapping symptomatologies, and hence pathomechanisms, stepwise cluster formation for PDS has included gastric accommodation, gastroduodenal inflammation, visceral hypersensitivity, and mucosal protection, in addition to the effect on 5-HT receptors (important for nausea). Fourth order cluster evaluation for PDS did not largely differ from cluster evaluation for EPS ( $\vee$ Fig. 5, Table 2S, Supporting Information). STW 5 was the most active preparation, followed in the same category $(++)$ by angelica > chamomile, greater celandine, liquorice $>$ and melissa. Less active $(+)$ were bitter candytuft, caraway $>$ milk thistle $>$ and peppermint ( $\bullet$ Fig. 5B).

\section{Irritable bowel syndrome}

IBS is a multifactorial disease with poorly understood pathophysiology. A clear link between possible pathophysiology and IBS symptoms is yet to be established.

Fourth order cluster analysis considers subsequent underlying pathologies: colon motility with the sub-clusters tonic effect, colon contraction, colonic neurotransmission, and spasmolytic effects. Additionally there are visceral hypersensitivity, inflammation, secretion, and defects in intestinal barrier. No specific data were available for STW 5's effects on intestinal microbiome and gas formation.

Based on the available pharmacological investigations concerning pathologies of IBS, fourth order cluster analysis further confirmed highest activity for STW $5(++)$. All contributing single plant extracts showed weaker effects, falling into the lowest effect size $(+)$, with bitter candy tuft, chamomile, and milk thistle being most active, followed by angelica and melissa ( $\bullet$ Fig. 6B). There may be several reasons for reduced effect sizes of STW 5's single constituents in IBS. One major aspect is the heterogeneity of the pathophysiological puzzlestones in IBS. This is a major argument supporting the use of combination drugs like STW 5 in the treatment of multifactorial diseases like IBS. STW 5 offers a mixture of active ingredients, granting individual pharmacological activities resulting in combined, superior clinical effects. This heterogeneity increases the likelihood of single plant extracts being inactive in counteracting certain pathophysiologies, as can be seen in colonic neurotransmission (Table 35, Supporting Information). Only bitter candytuft resulted in an increase of neurotransmission (which fits to its tonicizing effects), while angelica root, melissa leaf, and greater celandine resulted in a decrease. However, it has to be emphasized that both tonic and spasmolytic effects of STW 5 (partly mediated through its effects on neurotransmission) may be helpful in relieving patients' symptoms, depending on the kind of complaints the individual patient suffers from.

Overall, though contribution to the sum effect of STW 5 of single plant extracts is low, fourth order cluster analysis resulted in effect sizes ranging from 33 to $66 \%$. This essential finding of our analysis holds true for the extremely broad range of pharmacological tests in which STW 5 has been investigated. This serves as solid platform for the already confirmed clinical efficacy of the extract combination in STW 5 in the treatment of IBS and helps us to understand that the composition STW 5 is the most powerful way to target patients' symptoms, since no individual extract exerts an effect overall comparable to the STW 5 summation effect. Our analysis was not able to identify the STW 5 effect as just being an addition of the individual plant extract effects; the overall effect is rather constituted by additive, super-additive, and multiplicity effects.

\section{Summary}

In summary, the reported innovative method of standardized categorization of effect sizes and subsequent stepwise cluster formation and cluster evaluation is a suitable tool to handle large and complex datasets. This method is therefore useful to summarize, quantify, and understand large datasets like the ones being available for phytotherapies with a multitude of mechanisms and sites of action. Based on the results, research gaps can be identified and possible underlying pathophysiological processes can be suggested, a feature that may advance scientific knowledge. Furthermore aspects on the mode of action of single plant extracts may become apparent. This method is not only suitable for combination preparations, but may also be extended to the level of extract fractions or single active compounds.

Using this innovative method, STW 5 was identified as a powerful preparation, helpful in the FGIDs IBS and FD, including both 
EPS and PDS. Whereas from a pharmacological point of view all single plant extracts show individual beneficial effects, from a medical point of view, in a combinatory drug all extracts contribute to a summation effect that may be higher than the individual effects. This summation effect is the mechanism that makes herbal combination preparations like STW 5 superior to individual plant extracts.

\section{Supporting Information}

Detailed tables showing all categorization results for the different models and pathomechanisms are available as supporting information.

\section{Acknowledgements}

This paper is dedicated to Professor Dr. Max Wichtl in recognition of his outstanding contribution to pharmacognosy research.

\section{Conflict of Interest}

HA an OK are fully employed by Steigerwald Arzneimittelwerk $\mathrm{GmbH}$. MS and GL received consultancy fees from the same company.

\section{References}

[1] Allescher HD. Functional dyspepsia - a multicausal disease and its therapy. Phytomedicine 2006; 13 (Suppl. 5): 2-11

[2] Rösch W, Liebregts T, Gundermann KJ, Vinson B, Holtmann G. Phytotherapy for functional dyspepsia: a review of the clinical evidence for the herbal preparation STW 5. Phytomedicine 2006; 13 (Suppl. 5): 114121

[3] Drossman DA. Functional gastrointestinal disorders: history, pathophysiology, clinical features, and Rome IV. Gastroenterology 2016; 150: 1262-1279

[4] Sunderland R. Irritable bowel syndrome in adults: symptoms, treatment and management. Nurs Stand 2017; 31: 52-63

[5] Enck P, Aziz Q, Barbara G, Farmer AD, Fukudo S, Mayer EA, Niesler B, Quigley EM, Rajilić-Stojanović M, Schemann M, Schwille-Kiuntke J, Simren M, Zipfel S, Spiller RC. Irritable bowel syndrome. Nat Rev Dis Primers 2016; 2: 16014

[6] Schoenfeld PS. Advances in IBS 2016: A review of current and emerging data. Gastroenterol Hepatol (N Y) 2016; 12 (Suppl. 3): 1-11

[7] Andresen V, Keller J, Pehl C, Schemann M, Preiss J, Layer P. Irritable bowel syndrome - the main recommendations. Dtsch Ärztebl Int 2011; 108 : 751-760

[8] Tack J, Talley NJ. Functional dyspepsia: symptoms, definitions and validity of the Rome III criteria. Nat Rev Gastroenterol Hepatol 2013; 10: 134141

[9] Rhee PL, Kim YH, Son HJ, Kim J], Koh KC, Paik SW, Rhee JC, Choi KW. The etiologic role of gastric hypersensitivity in functional dyspepsia in Korea. J Clin Gastroenterol 1999; 29: 332-335

[10] Talley NJ, Walker MM, Holtmann G. Functional dyspepsia. Curr Opin Gastroenterol 2016; 32: 467-473

[11] Talley NJ, Ford AC. Functional dyspepsia. N Engl J Med 2015; 373: $1853-$ 1863

[12] Wegener $\mathrm{T}$, Wagner $\mathrm{H}$. The active components and the pharmacological multi-target principle of STW 5 (Iberogast). Phytomedicine 2006; 13 (Suppl. 5): 20-35
[13] Hohenester B, Rühl A, Kelber O, Schemann M. The herbal preparation STW 5 (Iberogast) has potent and region-specific effects on gastric motility. Neurogastroenterol Motil 2004; 16: 765-773

[14] Schemann M, Michel K, Zeller F, Hohenester B, Rühl A. Region-specific effects of STW 5 (Iberogast) and its components in gastric fundus, corpus and antrum. Phytomedicine 2006; 13 (Suppl. 5): 90-99

[15] Khayyal MT, el-Ghazaly MA, Kenawy SA, Seif-el-Nasr M, Mahran LG, Kafafi YA, Okpanyi SN. Antiulcerogenic effect of some gastrointestinally acting plant extracts and their combination. Drug Res 2001; 51: 545553

[16] Khayyal MT, Seif-el-Nasr M, El-Ghazaly MA, Okpanyi SN, Kelber O, Weiser D. Mechanisms involved in the gastro-protective effect of STW 5 (Iberogast) and its components against ulcers and rebound acidity. Phytomedicine 2006; 13 (Suppl. 5): 56-66

[17] Beil W. In vitro Untersuchungen mit Iberogast. Study Report 13. Institut für Allgemeine Pharmakologie, Medizinische Hochschule Hannover. Darmstadt: Dokumentation Steigerwald; 1999

[18] Schemann M, Angay O, Wagner S, Krueger D, Zeller F, Kelber O. Wirkung von STW 5 auf die Motilität des unteren Oesophagussphinkters und des Kolons: neue Indikationen für Iberogast. Z Gastroenterol 2008; 46: 1039, P354

[19] Pilichiewicz AN, Horowitz M, Russo A, Maddox AF, Jones KL, Schemann M, Holtmann G, Feinle-Bisset C. Effects of Iberogast on proximal gastric volume, antropyloroduodenal motility and gastric emptying in healthy men. Am J Gastroenterol 2007; 102: 1276-1283

[20] Abdel-Aziz H, Zaki HF, Neuhuber W, Kelber O, Weiser D, Khayyal MT. Effect of an herbal preparation, STW 5, in an acute model of reflux oesophagitis in rats. J Pharmacol Sci 2010; 113: 134-142

[21] Holtmann G. Talley NJ. Functional dyspepsia. Curr Opin Gastroenterol 2015; 31: 492-498

[22] Abdel-Aziz H, Schneider M, Neuhuber W, Kassem AM, Khailah S, Müller ], Gamaleldeen H, Khairy A, Khayyal MT, Shcherbakova A, Efferth T, UlrichMerzenich G. GPR84 and TREM-1 signaling contribute to the pathogenesis of reflux esophagitis. Mol Med 2015; 21: 1011-1024

[23] Michael S, Kelber O, Hauschildt S, Spanel-Borowski K, Nieber K. Inhibition of inflammation-induced alterations in rat small intestine by the herbal preparations STW 5 and STW 6. Phytomedicine 2009; 16: 161171

[24] Michael S, Abdel-Aziz H, Weiser D, Müller CE, Kelber O, Nieber K. Adenosine $\mathrm{A}(2 \mathrm{~A})$ receptor contributes to the anti-inflammatory effect of the fixed herbal combination STW 5 (Iberogast) in rat small intestinal preparations. Naunyn Schmiedebergs Arch Pharmacol 2012; 385: 411421

[25] Nieber K, Voß U, Abdel-Aziz H, Kelber O, Weiser D. Geschlechtsspezifische Untersuchungen zum Einfluss von STW 5 auf Entzündungsprozesse an Dickdarmpräparaten. Z Gastroenterol 2013; 51: 928, K185

[26] Voß U, Michael S, Kelber O, Weiser D, Nieber K. Untersuchungen zur Wirkung von STW 5 und STW 6 auf den Tonus und die Kontraktilität an Dickdarmpräparaten Ratte. Z Gastroenterol 2011; 49: 1090, P2.136

[27] Voß U, Herr F, Störmer N, Okpanyi S, Weiser D, Kelber O, Abdel-Aziz H, Nieber K. Effects of STW 5, STW 5II and their components on contractility of inflamed rat colon preparations. Young Researchers Meeting. Münster, 2012

[28] Bonaterra GA, Kinscherf R, Kelber O, Weiser D, Metz ]. Antiinflammatorische und antiproliferative Wirkung von Iberogast in vitro. Z Gastroenterol 2008; 46: 1038, P352

[29] Bonaterra GA, Kelber O, Weiser D, Kinscherf R. Mechanisms of the antiproliferative and anti-inflammatory effects of the herbal fixed combination STW 5 (Iberogast) on colon adenocarcinoma (HT29) cells in vitro. Phytomedicine 2013; 20: 691-698

[30] Marx L, Grundmann D, Schreiber D, Klotz M, Simon D, Rabe H, AbdelAziz H, Kelber O, Schäfer KH. Intestinal inflammation and motility: how does STW 5 influence this? Z Phytother 2016; 37 (Suppl. 1): S46 
[31] Schneider M, Efferth T, Abdel-Aziz H. Anti-inflammatory effects of herbal preparations STW 5 and STW 5-II in cytokine-challenged normal human colon cells. Front Pharmacol 2016; 7: 393

[32] Wadie W, Abdel-Aziz H, Zaki HF, Kelber O, Weiser D, Khayyal MT. STW 5 is effective in dextran sulfate sodium-induced colitis in rats. Int J Colorectal Dis 2012; 27: 1445-1453

[33] Khayyal MT, Wadie W, Abdallah D, Kelber O, Abdel-Aziz H. Underlying mechanisms involved in the potential usefulness of the herbal preparation STW 5 in an experimental model of Crohn's disease. Planta Med 2016; 81: S1-S381

[34] Schempp H, Toth A, Weiser D, Elstner EF. Mean-antioxidative properties of Iberis amara extracts in biochemical model reactions. Drug Res 2003; 53: 568-577

[35] Germann I, Hagelauer D, Kelber O, Vinson B, Laufer S, Weiser D, Heinle $\mathrm{H}$. Antioxidative properties of the gastrointestinal phytopharmaceutical remedy STW 5 (Iberogast). Phytomedicine 2006; 13 (Suppl. 5): 45-50

[36] Liu CY, Müller MH, Glatzle J, Weiser D, Kelber O, Enck P, Grundy D, Kreis ME. The herbal preparation STW 5 (Iberogast) desensitizes intestinal afferents in the rat small intestine. Neurogastroenterol Motil 2004; 16 : 759-764

[37] Müller MH, Liu CY, Glatzle J, Weiser D, Kelber O, Enck P, Grundy D, Kreis ME. STW 5 (Iberogast) reduces afferent sensitivity in the rat small intestine. Phytomedicine 2006; 13 (Suppl. 5): 100-106

[38] Mittler S, Müller MH, Kasparek MS, Kelber O, Weiser D, Kreis ME. Iberis amara desensitizes colonic afferent nerve fibers in the mouse to 5-HT and low-threshold distension but not to bradykinin and high-threshold distension. Gastroenterology 2011; 140: S537
[39] Simmen U, Kelber O, Okpanyi SN, Jaeggi R, Bueter B, Weiser D. Binding of STW 5 (Iberogast) and its components to intestinal 5-HT, muscarinic M3, and opioid receptors. Phytomedicine 2006; 13 (Suppl. 5): 51-55

[40] Abdel-Aziz H, Kelber O, Vinson B, Okpanyi SN, Khayyal MT. STW 5 in a model of 5-HT3 receptor mediated in vitro. Z Gastroenterol 2007; 45: 767, P079

[41] Ammon HPT, Kelber O, Okpanyi SN. Spasmolytic and tonic effect of Iberogast (STW 5) in intstinal smooth muscle. Phytomedicine 2006; 13 (Suppl. 5): 67-74

[42] Voß U, Michael S, Kelber O, Weiser D, Nieber K. Vergleichende Untersuchungen zur Wirkung von STW 5 und STW 6 auf den Tonus und die Kontraktilität an Dünn- und Dickdarmpräparaten Ratte. Z Gastroenterol 2010; 48: 955, P265

[43] Allam S. Actions of the phytopharmacon Iberogast in different regions of the gut [dissertation]. Munich: Technical University; 2014

[44] Sibaev A, Abdel-Aziz H, Kelber O, Allescher HD, Storr M. Mechanism of action of STW 5 and its components on motility and intestinal neurotransmission in "in vitro" model of the human colon. Neurogastroenterol Motil 2015; 27 (Suppl. 2): 101

[45] Allam S, Krueger D, Demir IE, Ceyhan G, Zeller F, Schemann M. Extracts from peppermint leaves, lemon balm leaves and in particular angelica roots mimic the pro-secretory action of the herbal preparation STW 5 in the human intestine. Phytomedicine 2015; 22: 1063-1070

[46] Lee KJ, Tack J. Duodenal implications in the pathophysiology of functional dyspepsia. J Neurogastroenterol Motil 2010; 16: 251-257 